\title{
Metastatic glioblastoma multiforme: A rare case of long-term survival.
}

\author{
Huaming Xiao", Jianfeng Bai ${ }^{1}$, Mingbu Yan${ }^{1}$, Kai Ji ${ }^{1}$, Wei Tian ${ }^{1}$, Dagang Liu ${ }^{1}$, Tongbo Ning ${ }^{1}$, Junyang \\ Kang $^{2}$, Jidian Zou ${ }^{*}$ \\ ${ }^{1}$ Department of Neurosurgery, Weihai Central Hospital, Weihai, Shandong, PR China \\ ${ }^{2}$ Department of Intensive Care Unit, Weihai Central Hospital, Weihai, Shandong, PR China
}

\begin{abstract}
Glioblastoma Multiforme (GBM) is the most common primary malignant brain tumor. Median survival is usually less than 1 year from the time of diagnosis, and even in the most favorable situations, a majority of patients die within 2 years. Standard therapy consists of surgical resection, radiotherapy and chemotherapy. In this case report, we described a 38 y old woman with GBM survived 7 years after a series of treatments. For the patient, the tumor was found extensively infiltrate the sagittal sinus, the surgery unable completely remove of tumor, radiotherapy, chemotherapy and individualized anti-cancer herbs therapy were further applied. After all therapy, the patient was awake, with moderate bone window tension, palpable mass, right partial hemianopia, good right limb muscle strength. In summary, comprehensive treatment schedule including thorough surgical removal of tumor, systematic radiation, standard chemotherapy and individualized anti-cancer herbs therapy can help to prolong the survival of patients with GBM and gliosarcoma.
\end{abstract}

Keywords: Glioblastoma multiforme, Metastasis, Survival time.

Accepted on December 12, 2017

\section{Introduction}

Glioblastoma Multiforme (GBM), as a grade IV astrocytoma defined by World Health Organization (WHO), represents the most common and aggressive form of primary malignant brain tumor in adults, with an incidence of 3.2 per 100,000 persons [1]. GBM has a complex biology and despite decades of research, remains unknown [2]. Recently, some reports of targeted therapy and immunotherapy have not been improvement the survival of patients with GBM [3]. Currently, patients usually have a median survival time of approximately 3 months without medical treatment [4]. Treatment in patients with a good performance status is multimodal with surgical resection, radiotherapy and chemotherapy [2,5]. However, in spite of the intensive treatment, patients have a poor prognosis, especially for elderly patients [6,7]. Treatment of recurrent GBM is challenging due to the limited efficacy of the therapeutic options and the lack of a stand care [3]. Herein, we presented a case of a $38 \mathrm{y}$ old woman who underwent a series of surgery, radiation and chemotherapy, and individualized anti-cancer herbs therapy in 7 years and still survive now.

\section{Case Report}

A 38 y old woman, due to acute intracerebral hemorrhage, underwent open brain surgery under general anesthesia when she was admitted to hospital in July 2008. Previously, the patient had a good health without any history of tumor and intracerebral hemorrhage. During operation, the hemorrhage and unclear boundary of the tumor lesion could be seen at brain temporal lobe. Histopathologically, the lesion at left temporal lobe was a GBM (WHO grade IV) (Figure 1). The patient had a good condition and could engage in normal work after discharge. In November 2009, re-examination of skull CT showed encephaledema, but it was not handled. In February 2010, head MRI showed that the left temporal lobe lesion presented inhomogeneous flower-ring enhancement, and nonintensified liquefied necrotic area in the center of lesion. Her condition was improved after radiation therapy.

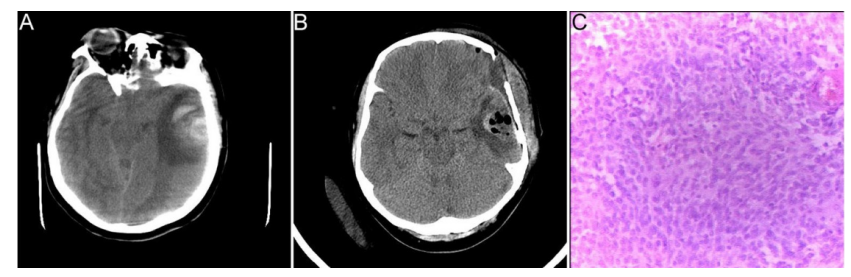

Figure 1. CT and pathological images was shown in the first surgery 2008. 7. Preoperative MRI showed high-density shadow around low density shadow in the left temporal lobe (a). Postoperative CT showed the hematoma was removal in the left temporal lobe (b). Postoperative pathological images showed large cell density, obvious special-shaped, common nuclear fission (c).

In June 2010, the patient underwent craniotomy for increased migraine headaches. The left temporal lobe brain tumor with partly liquefaction and yellowing in the surgery was resected. 
The pathology revealed classical GBM (Figure 2). The patient recovered to normal life due to traditional Chinese medicine decoction treatment (nightshade, selaginella, doederleinii hieron) after discharge.

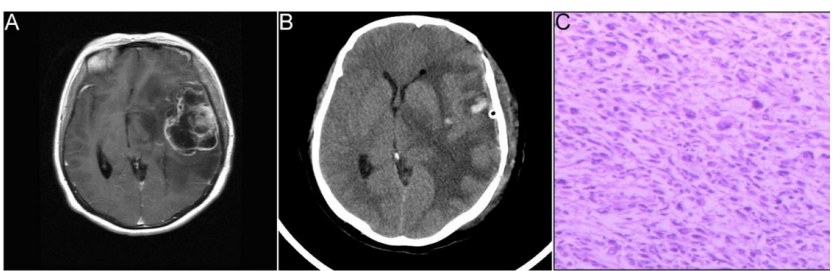

Figure 2. CT and pathological images was shown in the second surgery 2010.06. Postoperative MRI showed irregular mixed high and low density shadow in the left temporal lobe and basal ganglia region (a). Postoperative CT showed blood gas density shadow was shown in the subdural, the midline structure obviously moves to the right (b). Postoperative pathological images showed glial cell proliferation, cell fusiform or circular and visible tumor giant cells with hemorrhage necrosis (c).

In September 2014, the patient was admitted to hospital because of headache and weakness. Head MRI images showed intracranial space-occupying, which was considered as tumor recurrence (Figure 3). After surgical resection for occupying lesions, the pathology revealed classical GBM. Immunohistochemistry (IHC) showed S-100+, GFAP \pm , CK-, Ki-67 10\%. The patient was given the stable treatment after the surgery.

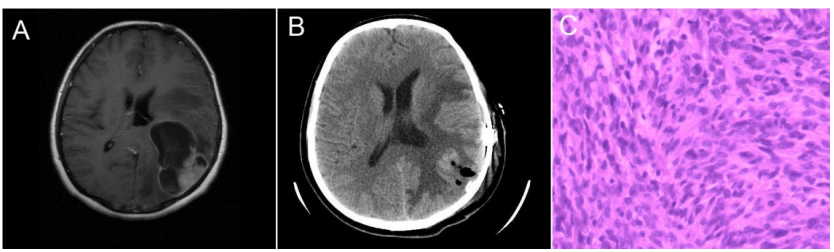

Figure 3. CT and pathological images was shown in the third surgery 2014.09. Preoperative MRI showed lesions narrow, the left parietal lobe of the abnormal ring and patchy. The condition improve that the midline structure moves to the right (a). Postoperative CT showed the lesions disappeared, the midline shift reduce (b). Postoperative pathological images showed cell fusiform or circular, special-shaped, common nuclear fission (c).

In January 2015, the patient was admitted to hospital because of headaches for $20 \mathrm{~d}$. MRI showed the tumor recurrence (Figure 4). Then, the patient was performed resection of left temporal and occipital tumor combined with bone flap decompression. From February 2015, radiotherapy (DT5000cGy/25f, 5f/w, 200cGy/f), carmustine (125 mg, 1-3 $\mathrm{d} / 3$ course), temozolomide ( $200 \mathrm{mg} / \mathrm{d}, 5 \mathrm{~d} /$ course) were performed to stabilize patient's condition.

In November 2015, the patient was admitted to hospital because of headache. The head MRI showed tumor recurrence. Postoperative pathology showed GBM recurrence that the tumor cells had invaded choroid plexus and skull. IHC showed Vimentin+, S-100+, Ki-67 5\%, EMA-, P53 10\%, GFAP-, CK-. After surgery, re-examination of the head MRI showed tumor recurrence, the tumor had invaded the sagittal sinus that could not for surgery (Figure 5). Hence, radiation and chemotherapy was performed. Until now, the patient is awake, with moderate bone window tension, palpable mass, right partial hemianopia, and good right limb muscle strength.

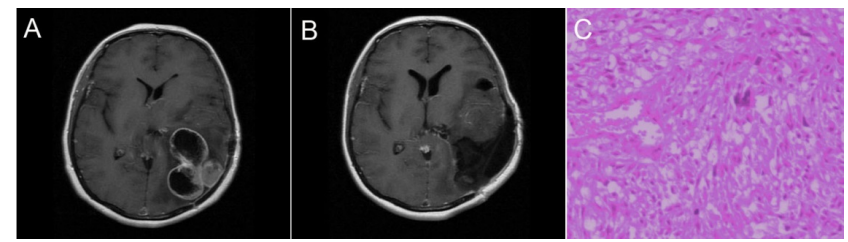

Figure 4. CT and pathological images was shown in the fourth surgery 2015.01. Preoperative brain MRI showed more lesions, more obvious strengthening signals, wall thickening, and edema is obvious surrounding lesions, the midline structure shift to the right (a). Postoperative CT showed, left skull is lost, irregular edema and a lot of gas density can be seen on the left side of brain, the midline structure shift to the right (b). Postoperative pathological images showed tumor cells arranged disorderly, special-shaped, common nuclear fission and necrosis (c).
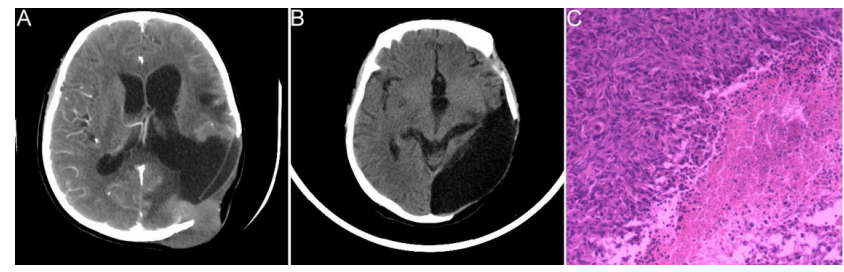

Figure 5. CT and pathological images was shown in the fifth surgery 2015.11. Preoperative brain MRI showed irregular shape abnormal signal, the boundary is clear, conform the recurrence of tumor (a). Preoperative brain CT showed tumor large and grow to extracranial (b). Postoperative pathological images showed tumor cells arranged disorderly, special-shaped, common nuclear fission and necrosis, Tumor cell invasion choroid plexus and skull, abundant cytoplasm (c).

\section{Discussion}

GBM is the most malignant astrocytic tumor in brain tumor, histopathologic lesion of GBM of grade IV defined by WHO [1]. For the GBM patients, the common therapies are microsurgery resection of lesions, radiotherapy and chemotherapy. In addition, calorie-restricted diet, psychological guidance and hyperbaric oxygen treatment can prolong the survival period of patients with GBM [8]. However, in spite of the intensive treatment, patients still have a poor prognosis with a median progression-free survival of 8.4 months, a median survival period of $16.8-21 \mathrm{w}$, and $5 \mathrm{y}$ overall survival of less than $10 \%$ [9]. In this study, the patient underwent a series of surgery resection, radiotherapy, chemotherapy, and individualized anti-cancer herbs therapy for 7 years is still alive.

GBM can influence the growth of the surrounding brain tissue by the invasion, so the total tumor cells were not cleared completely by the surgery. For GBM patients, postoperative MRI can show enhanced brain lesions, however, it is hard to identify the tumor recurrence or tumor progress. Therefore, there is no exact concept of recurrent GBM so far. Hsieh et al 
reported that for patients with GBM, recurrence in situ was common, multiple lesions or distant intracranial metastases accounted for 5\%. [10]. In this study, patient showed multiple lesions and mobility metastases, the primary lesions were at the left temporal lobe, gradually migrating to the rear, the temporal pillow direction, eventually reach the center line.

In this study, the pathology images of the patient showed that GBM and gliosarcoma coexist. Gliosarcoma is a rare malignant tumor of the primary central nervous system, it has components of both mesenchymal and glial differentiation. The therapy protocols are similar between GBM and gliosarcoma. There was no difference on clinical manifestation, which all showed increasing of intracranial pressure and focal neurological dysfunction. The difference of imaging showed that GBM can cross the midline and irregular flowers reinforcement, however, colloid sarcoma rarely across the midline, irregular concrete strengthening or irregular thick wall ring strengthening was presented. Imaging features of GBM need be differed from meningiomas and metastatic tumor. Meningiomas belong to the lesions outside the brain, local brain tissue edema was also significant. Metastatic tumor usually occurred in old men, which was located at junctional zone between ectocinerea and alba of cerebrum, tumor volume is small, but accompanied by severe edema around the tumor tissue. Castelli et al. reported that survival period of patients with gliosarcoma was 16.2 months after comprehensive therapy of surgery, radiotherapy and chemotherapy, two-year survival rate of patients with gliosarcoma was $12 \%$ after comprehensive therapy, the non-recurrence rate was only $2 \% 2$ $\mathrm{y}$ after surgery [11].

In this study, the tumor grew from intracranial to extracracranial, transferred from former to backward. GBM and gliosarcoma was comprehensive treated by surgical, radiation, chemotherapy and Chinese herbal medicine treatment. The reason which patient with tumor had long-term survival was included complete resection degree of the tumor, the speed of growth and aggressive, therapy of radiation, chemotherapy, and other treatment are in place. The herbs such like Morel, stone cypress, golden grass and so on had been used for treating tumor, the effect had been recognized [12]. The patient had experienced five surgical removal of the tumor, radiation and chemotherapy, simultaneous anti-cancer herbs therapy, resulted in the good effect.

\section{Conclusion}

Comprehensive schedule including thorough surgical removal of tumor, systematic radiation, standard chemotherapy and individualized Chinese herbal medicine treatment helps to prolonging the survival of GBM and gliosarcoma.

\section{Acknowledgements}

None

\section{Conflict of Interest}

\section{References}

1. Snyder J, Walbert T. Managing Glioblastoma in the Elderly Patient: New Opportunities. Oncology (Williston Park) 2017; 31: 476-483.

2. Noroxe DS, Poulsen HS, Lassen U. Hallmarks of glioblastoma: a systematic review. ESMO Open 2016; 1: 000144.

3. Nam JY, de Groot JF. Treatment of glioblastoma. J Oncol Pract 2017; 13: 629-638.

4. Chaichana KL, Pendleton C, Chambless L, CamaraQuintana J, Nathan JK, Hassam-Malani L, Li G, Harsh GR, Thompson RC, Lim M. Multi-institutional validation of a preoperative scoring system which predicts survival for patients with glioblastoma. J Clin Neurosci 2013; 20: 1422-1426.

5. Faycal L, Mouna B, Najia EA. Rare case of conus medullaris glioblastoma multiforme in a teenager. Surg Neurol Int 2017; 8: 234.

6. Greer L, Pannullo SC, Smith AW, Taube S, Yondorf MZ, Parashar B, Trichter S, Nedialkova L, Sabbas A, Christos P, Wernicke AG. Accelerated hypofractionated radiotherapy in the era of concurrent temozolomide chemotherapy in elderly patients with glioblastoma multiforme. Cureus 2017; 9: e1388.

7. Wick A, Kessler T, Elia AEH, Winkler F, Batchelor TT, Platten M, Wick W. Glioblastoma in elderly patients: solid conclusions built on shifting sand? Neuro Oncol 2017.

8. Poff AM. Targeting cancer metabolism with ketosis and hyperbaric oxygen. Univ South Florida 2014.

9. Chinot OL, Wick W, Mason W, Henriksson R, Saran F, Nishikawa R, Carpentier AF, Hoang-Xuan K, Kavan P, Cernea D. Bevacizumab plus radiotherapy-temozolomide for newly diagnosed glioblastoma. N Engl J Med 2014; 370: 709-722.

10. Hsieh JK, Hong CS, Manjila S, Cohen ML, Lo S, Rogers L, Sloan AE. An IDH1-mutated primary gliosarcoma: case report. J Neurosurg 2016; 1-5.

11. Castelli J, Feuvret L, Haoming Q, Biau J, Jouglar E, Berger A, Truc G, Gutierrez FL, Morandi X, Le Reste P. Prognostic and therapeutic factors of gliosarcoma from a multi-institutional series. J Neuro-Oncol 2016; 1-8.

12. An L, Tang J, Liu X, Gao N. Review on morel antitumor mechanism. Hina J Chin Mater Med 2006; 31: 1225-1226.

\section{*Correspondence to}

Jidian Zou

Department of Neurosurgery

Weihai Central Hospital

PR China

Email: 1819123872@qq.com

None 